\title{
Hepatic stem cells and cancers: a pathologist's view
}

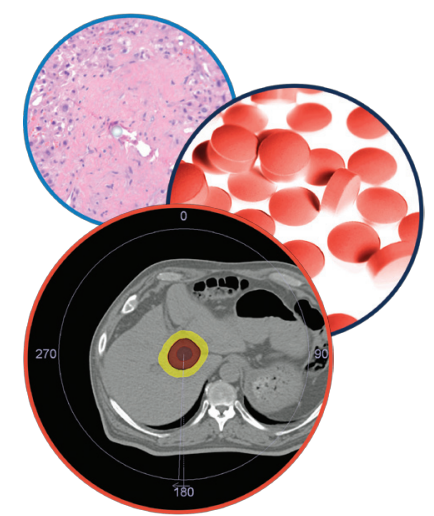

Hepatic Oncology

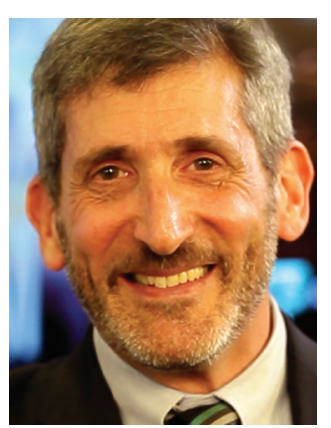

Neil Theise* speaks to Georgia Patey, Commissioning Editor: Neil Theise is a diagnostic liver pathologist, adult stem cell researcher and complexity theorist in New York City, where he is a Professor of Pathology at the Mount Sinai Beth Israel Medical Center of Icahn School of Medicine at Mount Sinai. He received his medical degree from Columbia University College of Physicians and Surgeons, where he also received his training in Anatomic Pathology. Subspecialty training was pursued in gastrointestinal (NYU), liver (Royal Free Hospital) and liver transplant (Mount Sinai, NYC) pathology. His earliest research focus was on defining the premalignant dysplastic nodules in human chronic liver disease. He revised understandings of human liver microanatomy, which in turn, led directly to identification of possible liver stem cell niches and the marrow-to-liver regeneration pathway. He is considered a pioneer of multiorgan adult stem cell plasticity. His publications on these topics in model systems and human liver stem cells have been highlighted on a record five covers of Hepatology.

\section{Q You are a diagnostic liver pathologist, adult stem cell researcher \& Professor of Pathology at the Mount Sinai Beth Israel Medical Center of Icahn School of Medicine. Can you tell our readers a little about your career to date? What would you class as your (\& your colleagues') greatest successes?}

There are relatively few specialists in liver pathology in the world compared with other fields of medicine in general, and pathology in particular, and we each prize our descent from the Founder, himself, Hans Popper. I can do so through three pupils/peers of his, each a master of liver pathology in their own right: P Scheuer (Royal Free Hospital), J Lefkowitch (Columbia College of Physicians and Surgeons) and S Thung (Icahn School of Medicine at Mount Sinai). Classically, as these mentors of mine have done, academic pathologists distribute their efforts equally among three sets of activities: clinical practice, teaching and scientific research; in the 21 st century, this means $60 \%$ clinical, $60 \%$ teaching and $60 \%$ research!

I would consider my own successes in each of these three realms to be equally important (and always in collaboration with clinical colleagues and other hepatopathology peers). I have helped to identify and define diagnostic criteria for human premalignant liver lesions (in particular, dysplastic nodules), new diagnostic criteria for primary biliary cirrhosis, and have helped to confirm the important role of ductular reactions in assessing human liver diseases. My interdisciplinary collaborations with radiologists and transplant surgeons to promote criteria and approaches to diagnostic screening for premalignancy and early

*Departments of Pathology \& Medicine (Division of Digestive Diseases), Mount Sinai Beth Israel Medical Center, First Avenue at 16th Street, New York, NY 10003, USA; ntheise@chpnet.org

\section{KEYWORDS}

- canals of Hering • cancer stem cells $\bullet$ combined hepatocellularcholangiocarcinoma

- hepatocellularcarcinoma • liver stem cells 
hepatocellular carcinoma (HCC) have been particularly satisfying. With regard to teaching, my editing and coauthoring of the most recent hepatobiliary chapters for the WHO Diagnostic Manual of Digestive Tract Tumors (the 'Blue Book') and authorship of liver chapters for Robbins Basic Pathology and Robbins and Cotran Pathologic Basis of Disease are perhaps culminating achievements, though daily liver pathology teaching of medical students, residents and fellows at the microscope remains a daily pleasure. In terms of science, redefining the microanatomy of the canal of Hering $(\mathrm{CoH})$ - the link between hepatocyte canaliculi and the biliary tree - is perhaps my biggest achievement [1]. Such a simple change in anatomy (that the $\mathrm{CoH}$ begins not at the limiting plate around the portal tract, but deeper in the hepatic parenchyma), has opened so many avenues of investigation. However one regards the importance of stem cells in liver functioning, this anatomic definition revealed the structure to be the primary, facultative, stem cell niche in humans. It is largely the source of the ductular reactions which are a primary response to diverse human diseases, of prime diagnostic and pathobiological importance, and what this revealed about the presence of hepatobiliary stem cells in human livers may be my most important accomplishment. However, beyond the liver world, it also directly led me to work in adult stem cell plasticity, complexity theory applications to cell biology, integrative medicine, science/spirituality dialogues and, most recently, consciousness studies. Canals of Hering to universal consciousness? Yes, a direct chain of concepts, if we have a few hours to talk...

\section{Q You are considered a thought leader in} liver stem cell biology; can you tell our readers about your recent work in this area?

There is now general agreement that livers have hepatobiliary stem cells, though their roles in development, tissue maintenance and repair, and carcinogenesis remain controversial. We have known for a long time that hepatocytes themselves have stem cell-like behaviors, most notably, how they can show the full measure of parenchymal restitution after partial hepatectomy and after all but the most severe acute hepatitis. How stem cells activate and participate in more severe acute injury or in chronic injuries over time has always been the open question.

I think that the controversy stems, in part, from the focus of most investigators on animal models as the only lens through which they evaluate stem cell functions. But rodent models of liver injury and repair differ from human models in a very important way: as yet there is no true animal model for the chronic injury leading to cirrhosis. There are repetitive acute injuries, such as serial carbon tetrachloride insults, but no true corollary to the long-term vascular distortions, scarring, hepatocyte senescence and ductular reactions of human cirrhosis. And, in chronic human liver injury, when cirrhosis is established, often robust repopulation of hepatocytes, greater than $50 \%$, has been demonstrated by my own laboratory and collaborators and by the laboratories of Malcolm Alison and Sir Nicholas Wright $[2,3]$.

Lineage tracking is difficult in human livers, but not impossible. In this regard, Alison and Wright have used histochemical and molecular analysis of mutations of mitochondrial DNA encoding for cytochrome c oxidase to explore the role of stem cells in human chronic liver disease [3]. They demonstrated large scale repopulation of regenerative cirrhotic nodules from adjacent ductular reactions, that is, $\mathrm{CoH}$-derived, activated progenitor cells, in viral hepatitis, autoimmune hepatitis, alcoholic and nonalcoholic fatty liver disease and Wilson disease.

Our own data concerning hepatocytes with and without expression of epithelial cell adhesion molecule (EpCAM) in chronic liver disease support this concept [2]. EpCAM-positive hepatocytes deriving from their associated EpCAMpositive ductular reactions have long telomeres like the progenitor cells to which they are tethered. EpCAM-negative hepatocytes, lacking association with ductular reactions and having short telomeres, are derived from pre-existing hepatocytes (perhaps the recently identified Axin-2 positive 'hepatocyte stem cells' [4]). EpCAM-negative hepatocytes predominate in early chronic liver disease (in this case, we studied chronic hepatitis $\mathrm{B}$ and $\mathrm{C}$ ), but as chronic disease progresses, the EpCAM-positive, stem cell-derived hepatocytes increase to greater than $50 \%$ of the parenchyma. Our current work employs differential expression of hepatitis B surface antigen in cirrhosis for additional, true lineage tracking [Theise N, Unpublished $\left.D_{A T A}\right]$ and we find, again, robust repopulation from the stem cell compartment.

The questions remain: how do we explain the differences between the robust stem cell-derived parenchymal repopulation of late stage chronic human disease and the very low-level hepatocyte 
production from the $\mathrm{CoH}$ in acute injuries and in early chronic human injury? And is there an important, though subtle role for stem cellderived hepatocytes, even when the repopulation is quite low? If we expect liver stem cells to behave like stem cells of other organs, such as hematopoiesis, skin and digestive tract, for which the definition includes robust repopulation of tissue in maintenance and/or repair, then one must dismiss liver stem cells as largely of trivial importance in most circumstances. If one allows, however, that stem cell definitions for different organs may be different, then one is open to explore some intriguing new possibilities.

The answers to these questions may lie in the special nature of liver microanatomy [5]. The liver is different from all other epithelial organs, in that its basement membrane is discontinuous, enveloping the entire biliary tree, but ending abruptly with the $\mathrm{CoH}$; hepatocytes have no basement membrane, only the loosely aggregated fibers of the space of Disse. This is important because in every other organ, when stem and progenitor cells produce new tissue, they merely follow the basement membrane to accomplish repair. In the liver, hepatocytes, lacking such basement membrane, have no consistent track to follow, whether produced by other hepatocytes and needing to find their way to the bile ducts, or produced by the $\mathrm{CoH}$ and needing to recreate the hepatic lobule.

We posit, based on our label retaining cell studies in murine acetaminophen toxicity [6], that the 'peribiliary hepatocytes' ( $\mathrm{PBH})$, that is, those hepatocytes directly attached to the $\mathrm{CoH}$ on one side and to other hepatocytes on their other side(s) are a distinct hepatocyte subsets. It is these PBH that are largely repopulated by liver stem cells; as hepatocytes divide and produce the bulk of parenchymal restitution, the $\mathrm{PBH}$, produced by as little as a single stem/progenitor cell division, await the arrival of those new hepatocytes, which do not have a basement membrane to track along, presenting them with the cell surface molecular organization to link to the biliary tree. Thus, in most liver injury, if the stem cell niche is activated, its teleological 'purpose' is not to restore parenchyma, but to re-establish the link between the parenchyma and the biliary tree, without which there would be no functional liver.

In chronic human liver diseases, the situation changes because the $\mathrm{PBH}$ have not been required to divide again in the earlier stages of disease, as demonstrated by their long telomeres. So, as hepatocyte-derived hepatocytes, dividing and dividing for years or decades, become senescent, these 'younger' hepatocytes take over roles of parenchymal restitution and expand significantly to accomplish the task. This hypothesis fits all the current data and I and collaborators in Japan, Belgium, and Germany are working to explore it further in mouse models and in human livers. We hope to have robust, reportable data to support this concept in the coming year.

\section{Q What do people mean by cancer stem} cells? So many people refer to them but mean different things. Can you shed any light on this complex issue?

There are two ways in which the phrase 'cancer stem cells' is used. 'Cancer stem cells' may indicate either 'stem or progenitor cells from which some cancers derive' or 'cancer cells, regardless of origin, which have stem cell-like functions'. These are two different concepts that can be confusing to the inexpert listener when the meaning is not precisely defined.

Where human liver is concerned, with strong support from rodent studies, HCCs, cholangiocarcinomas and tumors with mixed differentiation, probably can derive from both mature hepatocytes, from cholangiocytes and from stem/progenitor cells, though the rates at which they do so is probably variable between different species and between different etiologic liver diseases or injury models [7-9].

Thus, while malignant transformation of hepatobiliary stem/progenitor cells may lead to some hepatobiliary malignancies, it is probably more useful to restrict the phrase 'cancer stem cell' to those malignant cells within a cancer that perform stem cell like functioning (whether developing these functions de novo during malignant transformation or maintaining them as progeny of transformed stem/progenitor cells). Such functions include resistance to ischemia, toxins, and radiation, that is, the functions that protect the stem cell niche in normal tissues, allowing them to perform their reparative functions, and in tumors in which they are responsible for resistance to antitumor therapy.

One of the aspects of cancer stem cells that also sometimes contribute to confusion is the temptation to insist that cancer stem cells can be perfect mimics of their normative counterparts. So, for example, stem cells in normal tissues are relatively few in number and relatively slow 
cycling. But in tumors, this may not be the case. A recent dramatic example comes from analysis of the fibrolamellar type of HCCs by the laboratory of Lola Reid, showing that most, if not all the tumor cells have a stem cell molecular profile and behavior [10].

\section{Q What are the main obstacles to overcome in translating clinical stem cell research from bench to bedside?}

The challenges to some extent derive from so much controversy, that is, do stem cells in the liver have a role to play that can be exploited? If they do not, then exploring the pathways whereby they act is an undervalued part of the current liver stem cell research scene. For example, in some animal research, stem cell factor, one of the modulating molecules in stem cell activation in murine and human livers, has been shown to ameliorate acetaminophen toxicity in mice. Other signaling pathways have been shown to play a role in functioning of the liver stem cell niche. There could perhaps be more done to exploit these signaling molecules as medical approaches to boosting endogenous stem cell functions in an acute disease.

If one is talking about isolating and expanding stem cells ex vivo in order to supply them in great numbers for tissue repair, the big question becomes which is the best source to utilize? There are so many possible cell types of interest. Some groups have focused on human fetal stem cells. Others focus on adult hepatobiliary stem cells, but these may also derive from multiple sites in the liver, for example the $\mathrm{CoH}$ or, also, the peribiliary glands around the largest intrahepatic ducts and the extrahepatic ducts. Other adult stem cell populations exist that can show hepatobiliary differentiation under the right conditions, such as hematopoietic stem cells and mesenchymal stem cells (from diverse tissues). Then, there are hepatobiliary cells of various stages of differentiation/maturation from induced pleuripotent stem cells. With much of today's research still resting on defining the 'best' populations to use that it sometimes seems difficult to get to the actual applications.

The question of how best to apply them likewise has a plethora of creative strategies. There is the potential, as noted, for clinical application of stem cell modulators to infusion of stem cells themselves, either into vascular compartments or directly into the liver, for building of artificial organs using stem cells to populate an engineered or decellularized, previously existing (human or other) liver [11].

The diversity of approaches is both exciting and hopeful, but it also to some extent limits the extension to clinical application as there is little unanimity to focus the field in a promising direction and collaboration among 'competitors' is rare. I remain convinced that such applications can and will work, but have less hope about how efficiently the field will, as a whole, move toward accomplishing any one of those possible interventions in the near term. Time will tell, of course. Perhaps we need a top-down, federally coordinated, 'Manhattan Project' for such efforts to foster collaboration over competition among investigators.

\section{Q Despite your ongoing basic research,} the foundation of your professional efforts remains your clinical \& consulting work. What, in your opinion, are the most intriguing diagnostic or therapeutic developments to have hit the world of liver cancer in the last few years?

I just returned from the annual meeting of the Laennec Hepatopathology Society in Paris; it is perhaps the largest formal annual gathering of academic liver pathologists (and some colleagues from other fields) in the world. A dominant theme is that all over the world, there is increasing appreciation for tumors with mixed hepatobiliary differentiation. This tumor class was highlighted in the recent edition of the WHO Blue Book in which, for the first time, these tumors had their own chapter. That served to catalyze a burst of interest and investigational activity [12]

It still remains uncertain whether mixed hepatobiliary tumors are merely better appreciated with our newer tissue diagnostic techniques (such as more sophisticated use of immunohistochemistry) or that they are in fact becoming more common. But, as data accumulates, the latter seems likely. Why might this be happening? Perhaps it is that prior to the last two decades there were no consistently successful treatments for most chronic liver diseases, particularly when they had gotten to later stages in which tumors could develop. But now, after decades of antiviral treatments, which often merely suppressed virus without eradication, these treatments may have changed the pathobiology - we do not really know if some of these treatments altered hepatocarcinogenesis even as 
the viruses were being targeted. There is also the rise of mixed viral infections or compound diseases (for example viral hepatitis mixed with alcoholic or nonalcholic fatty liver disease). There is also a phenomenon recognized in many liver transplant centers: the 'dedifferentiation' of partially treated $\mathrm{HCC}$ with emergence of biliary immunophenotypes in the tumors (e.g., keratin 19 positive) or tumor stem cell populations selected for their resistance to therapy or overt combined hepatocellular-cholangiocarcinomas.

Pretreatment diagnosis with current clinical approaches is difficult given that diagnosis of HCC still rests on purely radiologic grounds without requiring biopsy. Moreover, even if a tumor is biopsied, there is as yet no standard for biopsying different regions of a single tumor to look for heterogeneity of differentiation. This area is ripe for interdisciplinary collaborative research with teams of pathologists, radiologists, hepatologists and surgeons.

Finally, we have yet to fully define how much nonalcoholic fatty liver disease is going to be a premalignant condition and how often these are of mixed phenotypes. Moreover, unlike all other chronic liver diseases, which promote hepatocarcinogenesis, nonalcoholic fatty liver disease seems to accomplish it even when there is little or no hepatic scarring. In a disease of truly vast, epidemic proportions, in which people without advanced stage disease can develop cancer, how do we develop cost efficient screening programs? Current modalities are inadequate. Again, it will be interdisciplinary collaborations between all the stakeholders in hepatology that will provide the meaningful answers.

\section{Q How do you see the reduction of} hepatitis $C$ virus following the advent of the highly effective anti-hepatitis $C$ virus therapies affecting liver pathology in the medium-to-long term? What will happen to

\section{References}

1 Theise ND, Saxena R, Portmann BC et al. The canals of Hering and hepatic stem cells in humans. Hepatology 30, 1425-1433 (1999).

2 Yoon SM, Gerasimidou D, Kuwahara R et al. Epithelial cell adhesion molecule (EpCAM) marks hepatocytes newly derived from stem/progenitor cells in humans. Hepatology 53, 964-973 (2011)

3 Lin WR, Lim SN, McDonald SA et al. The histogenesis of regenerative nodules in human

those with cirrhosis? What can we expect to happen regarding risk for hepatocellular carcinoma?

This question is one of the most pressing in the new, 'post-hepatitis $\mathrm{C}$ virus (HCV)' era. There is already data from the interferon/ribavirin era to suggest that progression to HCC slows in patients treated with long-term elimination of virus, but it does not disappear altogether. We know the age old description of viral cirrhosis as a premalignant condition is wrong: hepatocarcinogenesis and progression to cirrhosis are parallel, not sequential processes, both of which usually take years to decades [13]. Eliminating the virus and allowing for regression of fibrosis does not eliminate epigenetic and genetic changes that eventually lead to malignancy. So many questions remain: is the risk of cancer diminished? By how much? Should people whose HCV infection has been truly cured be screened for carcinoma anyway? For how long? And by what methods? There is no hepatologist treating hepatitis $\mathrm{C}$ in the world who is not already thinking about these questions. Only time will allow us to accrue sufficient data to understand our best approaches.

\section{Disclaimer}

The opinions expressed in this interview are those of the interviewee and do not necessarily reflect the views of Future Medicine Ltd.

Financial \& competing interests disclosure

The author has no relevant affiliations or financial involvement with any organization or entity with a financial interest in or financial conflict with the subject matter or materials discussed in the manuscript. This includes employment, consultancies, honoraria, stock ownership or options, expert testimony, grants or patents received or pending, or royalties.

No writing assistance was utilized in the production of this manuscript.

liver cirrhosis. Hepatology 51, 1017-1026 (2011).

Zhoo L, Fish M et al. Self-renewing diploid Axin2(+) cells fuelhomeostatic renewal of the liver. Nature 524, 180-185 (2015).

5 Theise ND, Dollé L, Kuwahara R. Low hepatocyte repopulation from stem cells: a matter of hepato-biliary linkage not massive production. Gastroenterology 145, 253-254 (2013).

6 Kuwahara R, Kofman AV, Landis CS, Swenson ES, Barendswaard E, Theise ND.
The hepatic stem cell niche: identification by label retaining cell assay. Hepatology 47, 1994-2002 (2008).

7 Holczbauer A, Factor VM, Andersen JB et al. Modeling pathogenesis of primary liver cancer in lineage-specific mouse cell types. Gastroenterology 145, 221-231 (2013).

8 Zucman-Rossi J, Nault JC, Zender L. Primary liver carcinomas can originate from different cell types: a new level of complexity in hepatocarcinogenesis. Gastroenterology 145 , 53-55 (2013). 
9 Raggi C, Factor VM, Seo D et al. Epigenetic reprogramming modulates malignant properties of human liver cancer. Hepatology 59, 2251-2262 (2014).

10 Oikawa T, Waulthier E, Dinh TA et al. Model of fibrolamellar hepatocellular carcinomas reveals striking enrichment in cancer stem cells. Nat. Commun. 6, 8070 (2015).
11 Michalopoulos GK, Grompe M, Theise ND. Assessing the potential of induced liver regeneration. Nat. Med. 19, 1096-1097 (2013).

12 Brunt EM, Sempoux C, Paradis V, Theise ND. Biphenotypic (hepatobiliary) primary liver carcinomas: the work in progress. Hepat. Oncol. 2(3), 255-273 (2015).
13 International Consensus Group for Hepatocellular Neoplasia (ICGHN) Group. Pathologic diagnosis of early hepatocellular carcinoma; a report of the International Consensus Group for Hepatocellular Neoplasia. Hepatology 49, 658-664 (2009). 